\title{
From Denture to the Final Implant-Supported Prosthesis Using a Full-Digital Protocol: A Dental Technique
}

\author{
Francisco Fernando Todescan ${ }^{1}$, Marcos Masayuki Hayashi ${ }^{1}$, Luiz Roberto Giugni ${ }^{1}$, Marco Antonio Bottino ${ }^{2}$ \\ and João Paulo Mendes Tribst ${ }^{3, *}$ (1) \\ 1 Oral Implantology Course, Foundation for the Scientific and Technological Development of \\ Dentistry (FUNDECTO), São Paulo University (USP), São Paulo 05508-000, Brazil; \\ ftodescan@uol.com.br (F.F.T.); mhimplante@gmail.com (M.M.H.); lgiuni@uol.com.br (L.R.G.) \\ 2 Department of Dental Materials and Prosthodontics, São Paulo State University (UNESP), \\ São Paulo 12245-000, Brazil; marco.bottino@unesp.br \\ 3 Academic Centre for Dentistry Amsterdam (ACTA), Department of Dental Materials, University of \\ Amsterdam and Vrije Universiteit Amsterdam, 1081 LA Amsterdam, The Netherlands \\ * Correspondence: j.p.mendes.tribst@acta.nl
}

Citation: Todescan, F.F.; Hayashi, M.M.; Giugni, L.R.; Bottino, M.A.; Tribst, J.P.M. From Denture to the Final Implant-Supported Prosthesis Using a Full-Digital Protocol: A Dental Technique. Oral 2021, 1 , 332-339. https://doi.org/10.3390/ oral1040033

Academic Editor: Michele Cassetta

Received: 8 November 2021

Accepted: 26 November 2021

Published: 1 December 2021

Publisher's Note: MDPI stays neutral with regard to jurisdictional claims in published maps and institutional affiliations.

Copyright: (c) 2021 by the authors. Licensee MDPI, Basel, Switzerland. This article is an open access article distributed under the terms and conditions of the Creative Commons Attribution (CC BY) license (https:// creativecommons.org/licenses/by/ $4.0 /)$.

\begin{abstract}
Proper maxillomandibular relationship registration can be clinically challenging during a digital workflow in full-arch rehabilitations. This dental technique requires the manufacturing and use of a hybrid surgical guide custom scanning device, indicated to be used during implant placement surgery, in addition to being used to simultaneously register the maxillomandibular relationship and transfer the implants' 3D positioning, ensuring a fully digital workflow in full-arch implant-supported prosthesis rehabilitation. The sequence of steps presented here will allow dentists and dental technicians to conduct rehabilitations from denture to the final implant-supported prosthesis using a full-digital protocol, using a minimal quantity of intraoral devices and digital tools.
\end{abstract}

Keywords: prosthodontics; digital technology; computer-aided design; implant-supported denture

\section{Introduction}

Digital impressions with intraoral scanners in a digital workflow have been welldocumented in the literature as a suitable alternative to conventional impressions [1] In this sense, virtual impressions can be easily performed, reduce visits to the dental office, are comfortable to the patient, can record the oral tissues without pressure, allow the correction of errors, and reduce the influence of dental material properties such as expansion, shrinkage, and distortion [2,3].

Although intraoral scanners can be used to digitally capture edentulous arches, they are usually difficult to use for inexperienced clinicians [3-5]. Errors in capture can generate distortion in the digital model, which can compromise the accurate fabrication of the prosthesis due to the failure in precisely replicating the implants' three-dimensional positioning and their relationship to the surrounding tissues [6,7].

Therefore, not only is correct implant placement essential to improve esthetic outcomes, prosthesis manufacturing with a passive fit is also necessary to guarantee the long-term success of dental implants with the stability of peri-implant tissues [8].

The versatile applicability of cone-beam computed tomography (CBCT) associated with 3D imaging tools strongly modified implant rehabilitation and treatment planning. Using virtual planning of implants' positions while considering the oral anatomic structures and future prosthetic needs improved dentists' and dental technicians' communication [5,9].

Furthermore, implant placement with computer-guided surgery can increase implant survival rates and reduce treatment costs in comparison to free-hand implant placement protocols [10].

Despite the increasing popularity of a digital workflow, the challenging maxillomandibular relationship registration is still one of the major difficulties when using the 
intraoral scan technique for prosthesis fabrication $[2,4,6,11]$. Previous reports have been proposed for complete-arch rehabilitations, describing the use of a muco-supported surgical guide with occlusal registration and scan bodies to fabricate a definitive fixed restoration, as well as digitally transferring from interim to definitive restoration [12,13].

In addition, there are reports showing that the failing dentition of partially edentulous individuals may be used as an initial reference for stackable restrictive surgical guides during full-arch immediate implant placement. The stackable guide option derived from a digital workflow can be used to increase the predictability during immediate implant placement, as well as the immediate loading of provisional implant-supported fixed dental prostheses [14].

Therefore, the present technique aims to demonstrates how to combine digital impressions with accurate maxillomandibular relationship transference using a modified surgical guide for implant-supported full-arch rehabilitations.

\section{Materials and Methods}

Technique Description

1. Perform the patient's documentation including clinical extra- and intraoral photos in occlusion and smiling (Figure 1).
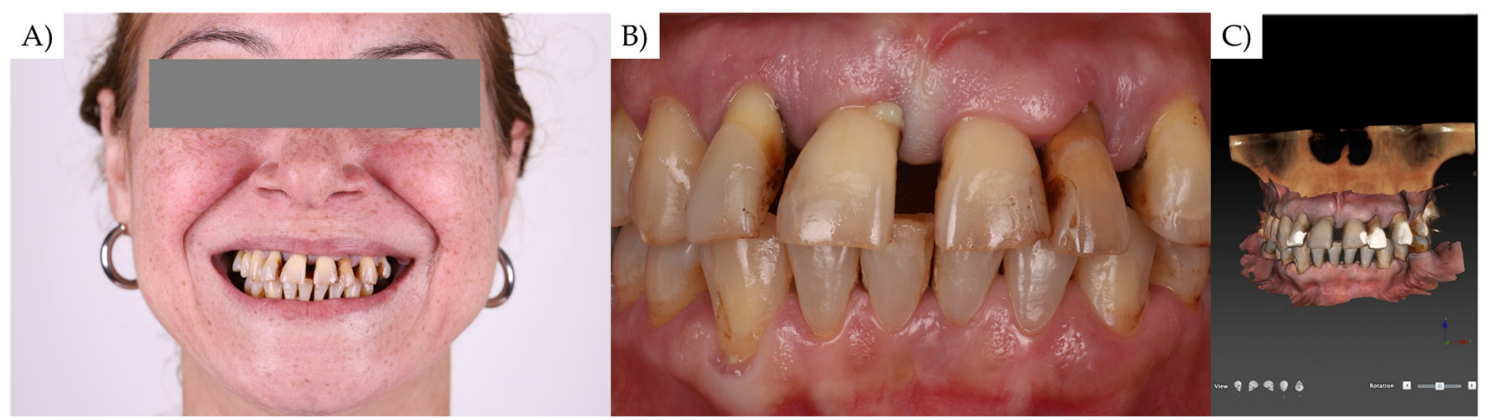

Figure 1. Complete documentation including face (A), smile, intra- (B) and extra-oral photographs, and intraoral scanning (C).

2. With the aid of an intraoral scanner device (Medit i500, Medit Corp., Seul, South Korea), perform a digital scan of the dentition (Figure 2). Before scanning, set up the order to export standard tessellation language (STL) files. Import the acquired digital data into CAD software (Dental System 2020; 3Shape Dental System; 3Shape A/S, Copenhagen, Denmark). With the aid of a modeling tool, perform a virtual extraction of the crowns and provide a new complete denture design with corrected esthetics and prosthetic outcomes following the workflow indicated by the software, and generate the new STL files.

3. Process the STL file of the new denture by using a 3D printer (5100 3D Systems; Nextdent) and denture resin (Denture 3D+; Nextdent). Follow the post-processing and finishing technique according to the manufacturer's instructions (Figure 3).

4. Follow the surgical plan, perform the necessary tooth extraction and install the printed denture during the healing period.

5. After healing, perform artificial landmarks using gutta-percha on the external surface of the buccal flange. Make a CBCT scan with and without the denture record in place (Figure 4). For this, the double tomography technique is used: first, the tomography is made with the patient wearing the denture with the gutta-percha marks in a seated position, then a second tomography is performed only for the prosthesis (outside the mouth). 


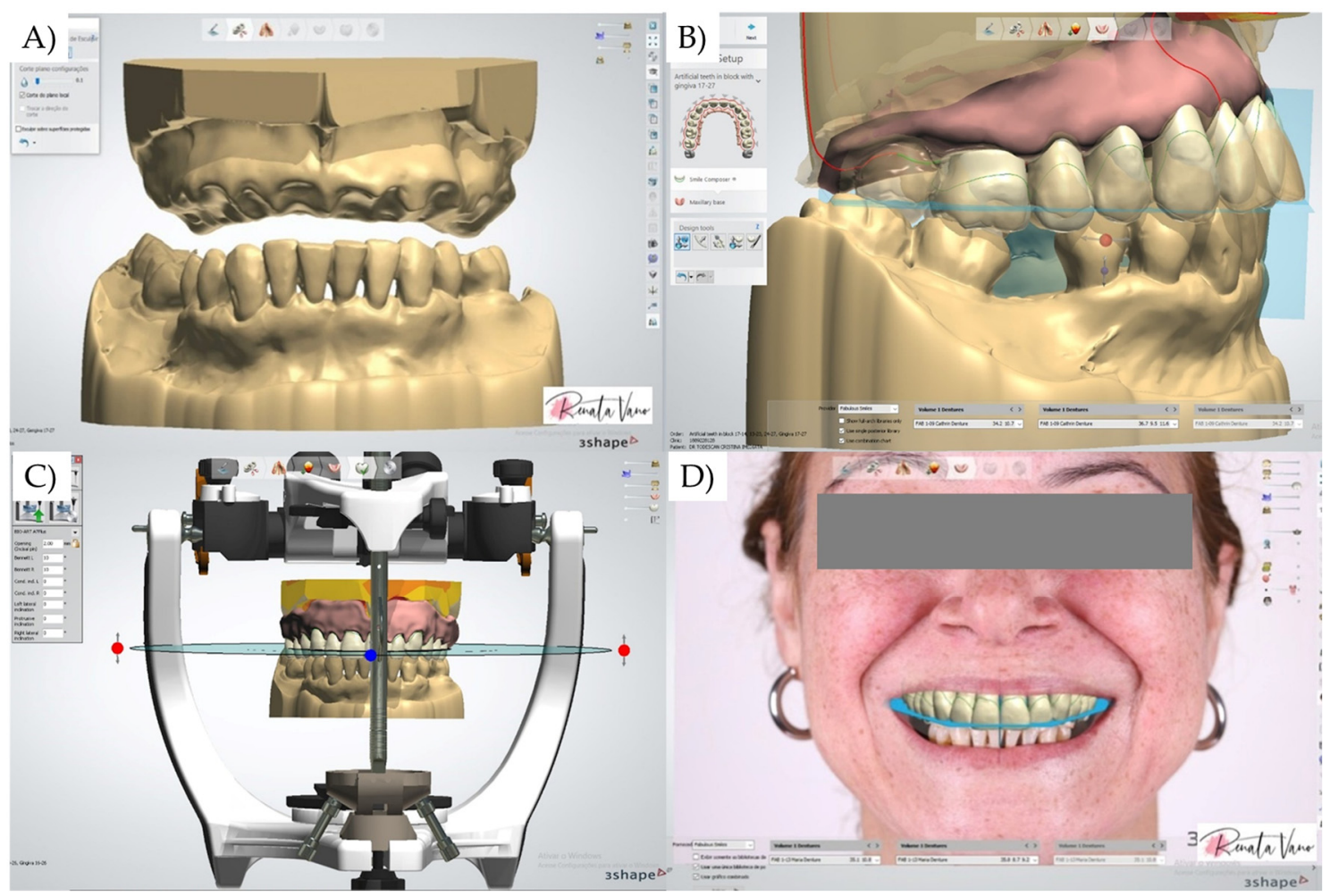

Figure 2. Data exported to CAD software, followed by the virtual extraction of all upper teeth (A). Virtual assembly of the artificial teeth in a new position (B). Correcting all the aesthetic and functional parameters using virtual articular (C,D).

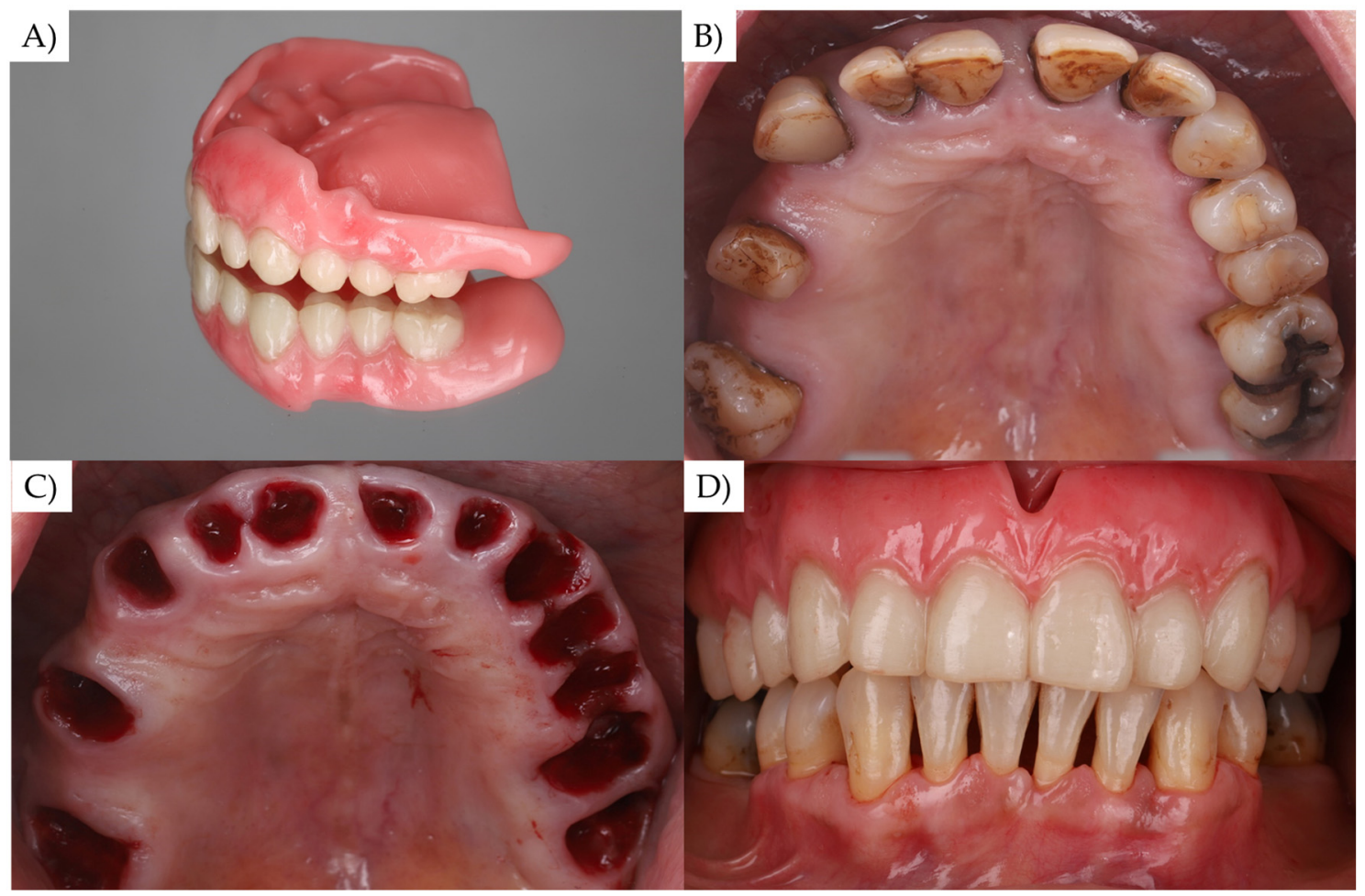

Figure 3. Three-dimensional-printed total denture designed in CAD (A), followed by extraction of upper teeth (B,C) and the installation as well as adjustment of the immediate denture for healing (D). 


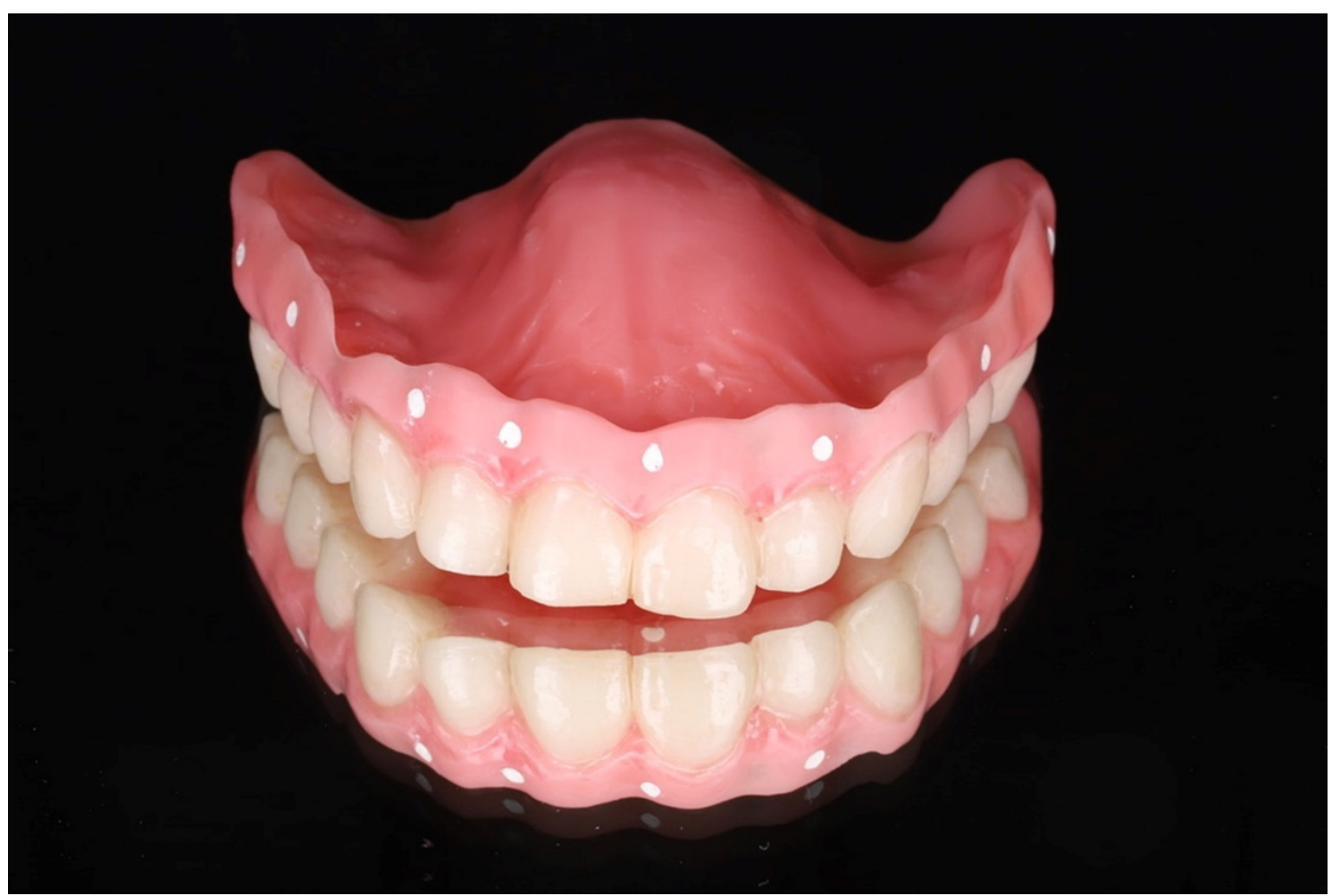

Figure 4. Artificial gutta-percha landmarks made on the complete denture, preparing for the image examination using tomography.

6. Identify at least 3 regions with positive and negative features that exhibit adequate contact intraorally. These areas need to be as far apart as possible (Figure 5).

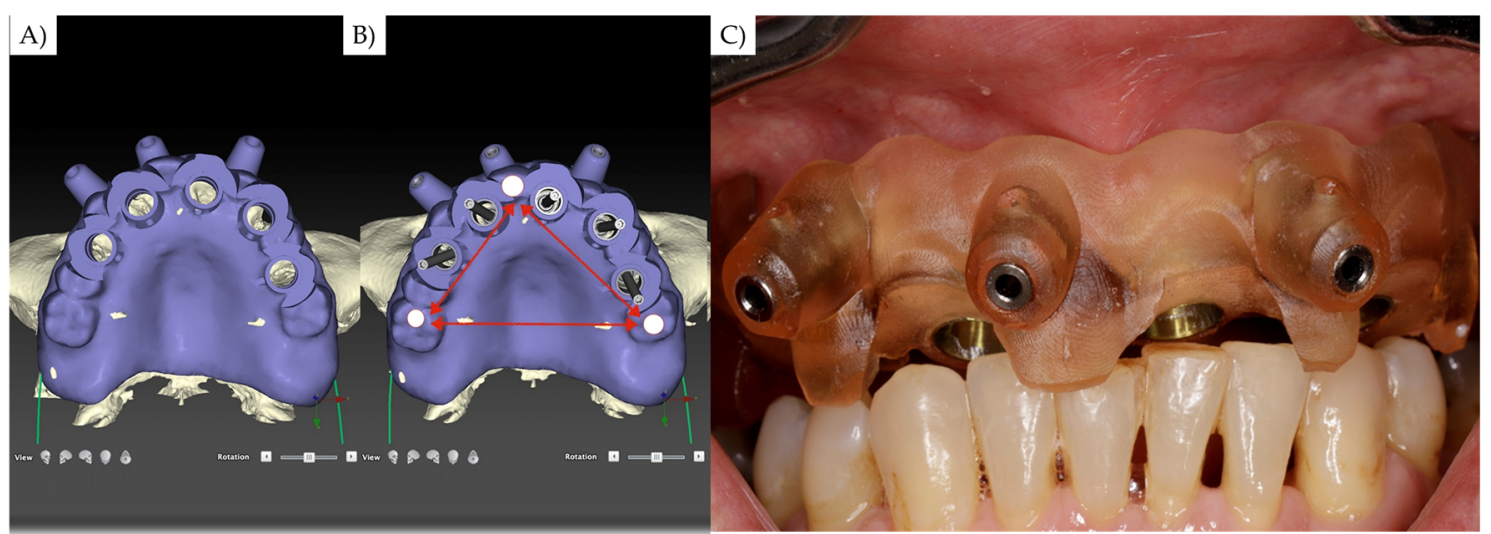

Figure 5. The 3D data were exported to the CAD software to plan the placement of the implants' prosthetic-driven position and the design of the hybrid surgical guide (A). Care was taken in the design of the device in order to maintain 3 points of occlusal contact, one in the anterior area and two in the posterior region, one on each side of the arch (B). This step will allow digital recording of the vertical dimension of occlusion, as well as the relationship between the arches, without the need to go through the analog phase (C). On the left, there is the occlusal view of the surgical guide obtained after planning 5 implants' placement in the ideal position from a biomechanical and aesthetic point of view, with reference to the complete denture. The image of the center shows how, in the design of the guide, 3 points of contact between the guide and the lower arch were maintained, in order to relate the upper and lower arches in a stable way, previously determined by the complete denture. Finally, the front view of the surgical guide in position, occluding with the lower arch in exactly the same digitally determined position. 
7. Import the DICOM file of the CBCT and the STL file of the digital scan of the dentures into the guided treatment planning software (DTX, KaVo Kerr, Brea, CA, USA) (Figure 6).

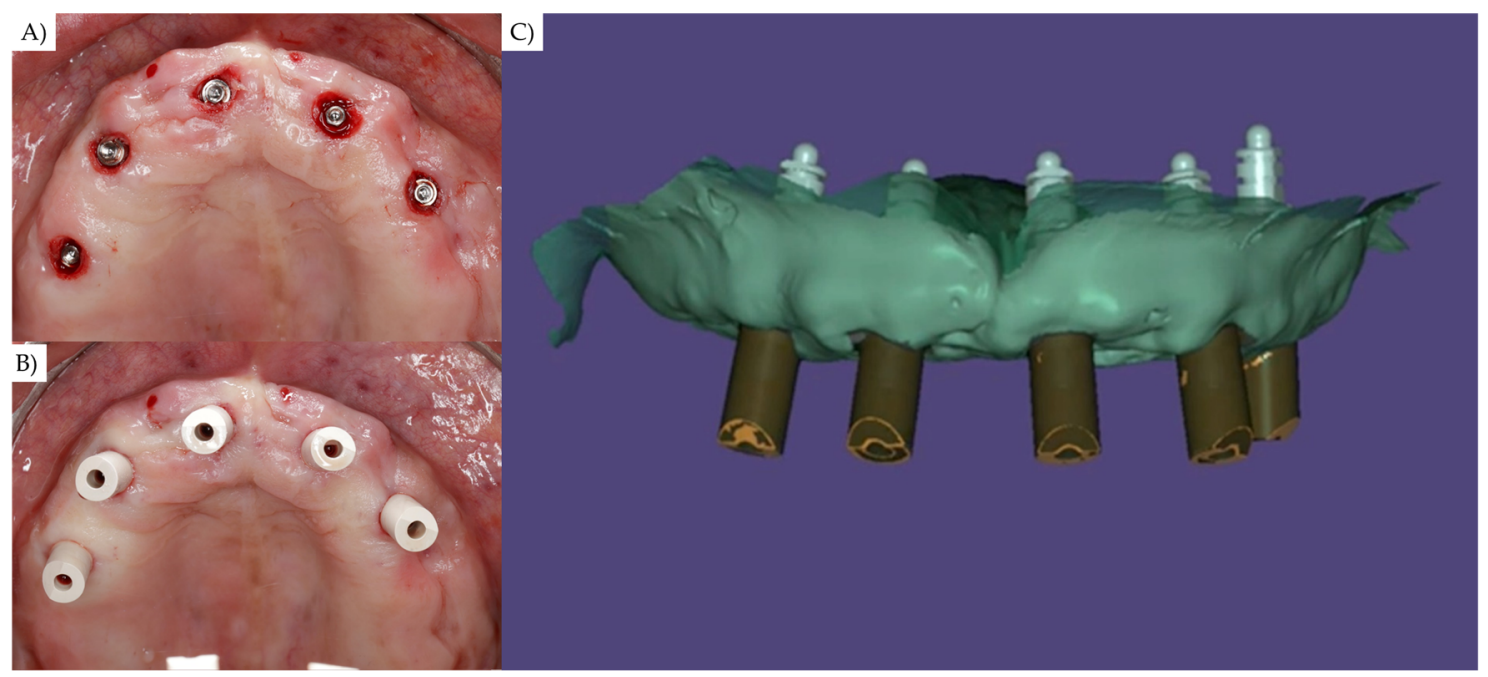

Figure 6. After the implants' placement (A) the first intraoral scan is performed using scan bodies (B,C).

8. Align the STL file with the DICOM file to obtain the relationship between the arches and denture. Then, plan the implants' 3D position according to your patient, individualizing the surgical guide with buccal screws to stabilize the device during the surgical procedure.

9. After the implants' placement, use a scan body in each implant to scan the arches (Medit i500, Medit Corp., Seul, South Korea) and the implants according to the manufacturer's instructions. Complete the scan and remove unnecessary areas (Figure 6).

10. Perform a second scan with a scan body and surgical guide placed in position. For this step, it is necessary to remove the restrictive metallic cylinders from the surgical guide. The surgical guide must by fixed by endosseuous fixation screws (Figure 7).
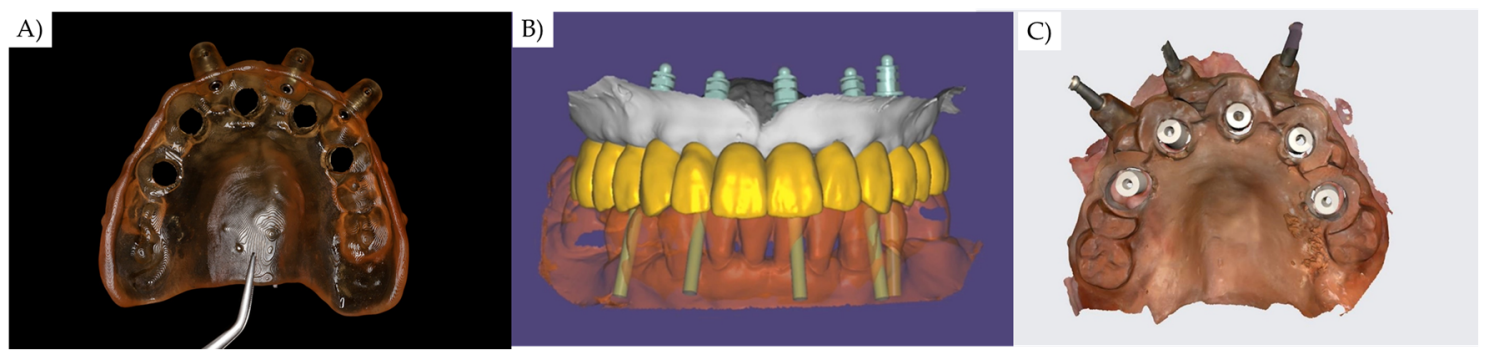

Figure 7. The second intraoral scan is performed with both the surgical guide and scan bodies in position. For that, the restrictive metallic cylinders should be removed from the guide, allowing it to fit in the previous surgical position (A). Then, it should be fixed with anchoring pins. After the second scanning, the clinical parameters can be transferred at the same time to the CAD software and aligned with the correct $3 \mathrm{D}$ position $(\mathrm{B}, \mathrm{C})$.

11. In the CAD software, align the antagonist arch scan with both STL file of the previous digital scan. This step will allow the recording of planned teeth position, 3D implants' positioning, and oral tissue characteristics, considering the maxillomandibular relationship and vertical dimension of occlusion (Figure 7).

12. Proceed with the prosthesis manufacturing based on the acquired information (Figure 8). 


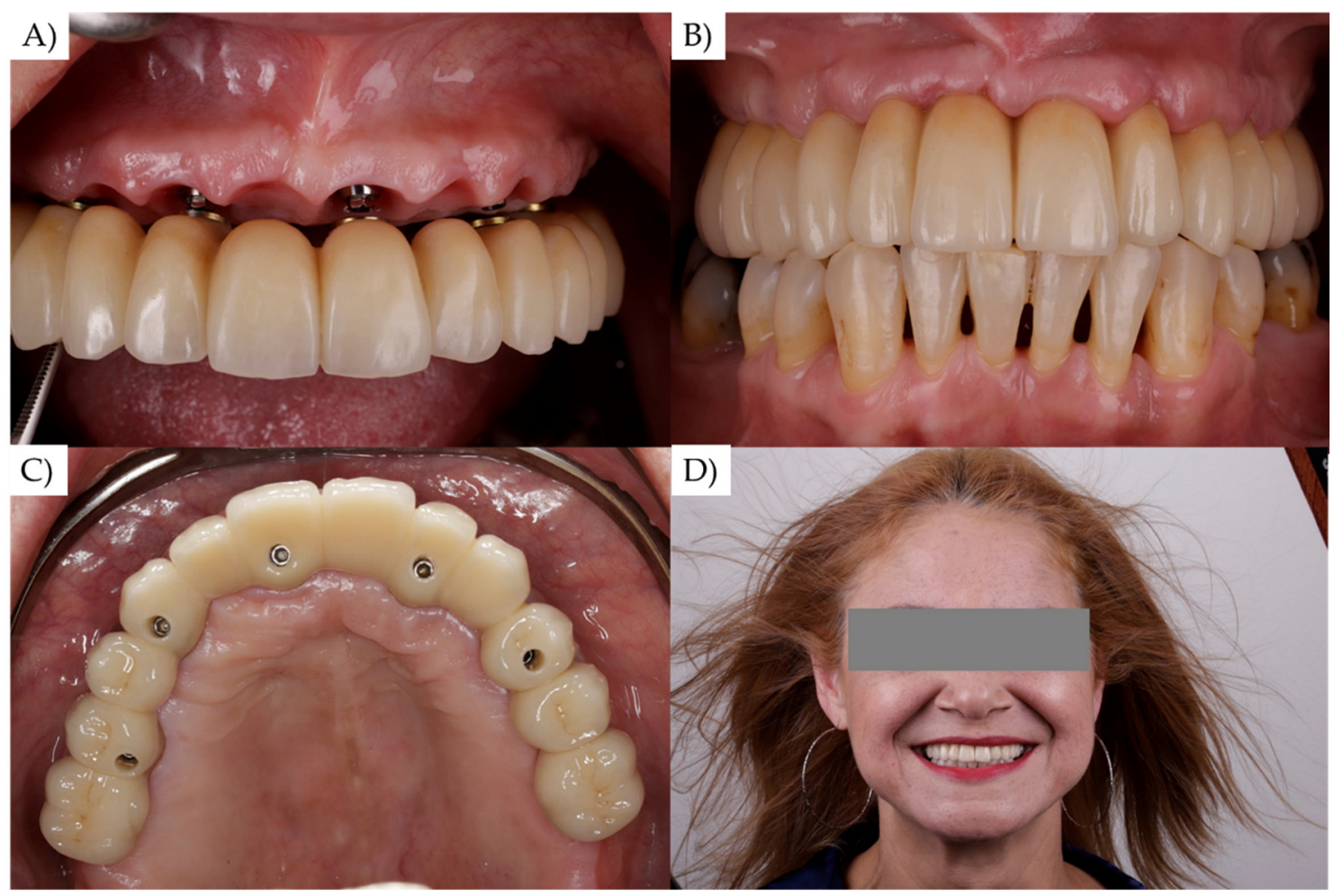

Figure 8. Following the informed data, the dental lab can proceed with the prosthesis manufacturing, respecting the esthetic and prosthetic outcomes. Final prosthesis installation (A), after occlusal adjustments (B), in occlusal view (C), and buccal view of the patient smile (D).

\section{Discussion}

The edentulous arch scan can be difficult, and viable techniques are not commonly described. Similar to previous reports [2,11,12], for the present dental technique, the manufacturing of a surgical guide requires an adequate, complete denture and knowledge of 3D modeling, CAD software, and 3D printer [2,11,12]. The use of a custom scanning device reduces the number of clinical steps, with adequate retention, stability, and support. However, in the present report the custom scanning device is a hybrid device applied in the surgical step for proper implant placement, as well as to improve the scanning process, the transferring of oral tissue characteristics, implants' position, and the maxillomandibular relationship registration.

Although the chair time can be reduced by following the described technical protocol $[2,13,14]$, it is necessary perform two scans instead of one [2]. Different from previous techniques, during the second scan, the surgical guide scanning device is indicated to be retained by endosseous screws that will guarantee a fixed retention, instead of just being muco-supported.

In general, surgical guides are easily adaptable to various clinical scenarios when they are properly supported. It has been shown that natural dentition and existing fixed dental prostheses provide support and stability to the guide during implant insertion [14]. The present study suggests the use of endosseus screws for that purpose.

The use of endosseus screws, working as anchoring pins, secured the surgical guide in the right position during the surgery and scanning process [15]. Generally, it is possible to observe three types of surgical guides (tooth-, mucosa-, and bone-supported). However, for full-arch rehabilitations only muco-supported and bone-supported can be considered, with the last one being suggested to reduce the risk of complications [16].

In addition to the mandatory knowledge, managing costs, as well as learning curve, it is necessary to use CAD software that allows the virtual alignment of similar 3D structures [11]. For that the software must present a friendly algorithm using the mathematical 
method to move the meshes into their "best fit" without overlapping between the STL files [2].

The traditional analogic workflow for implant-supported rehabilitations in an edentulous maxilla requires an existing denture to prosthetically drive the implant placement. Therefore, the presented approach does not use analogic processes as cast models or impression materials, but still need an existing denture for the similar aim of prosthetically driving the implant placement. The summarized workflow for each approach can be observed in the flowchart from Figure 9.
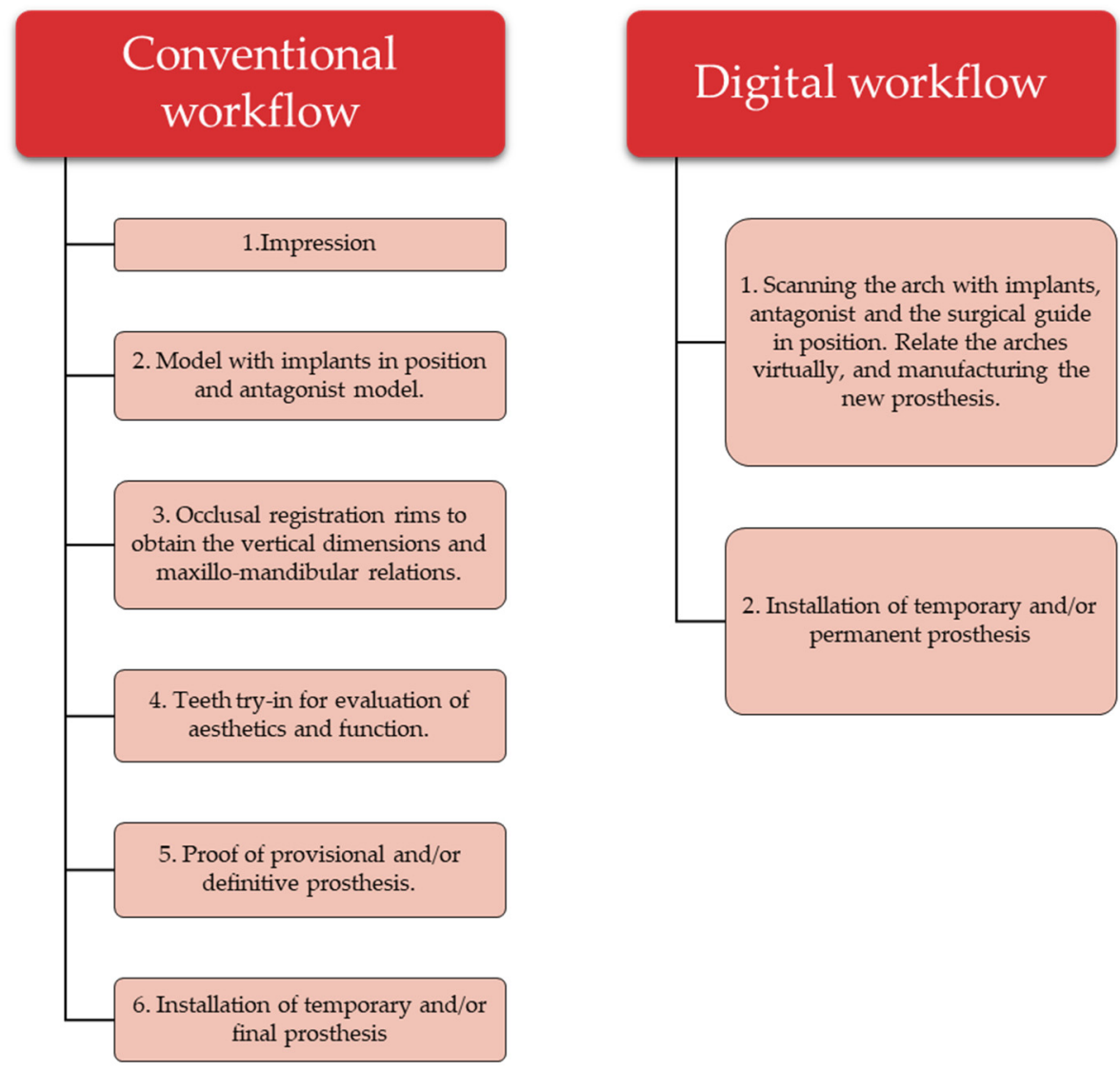

Figure 9. Flowchart for the conventional and digital workflows with the major clinical steps that should be considered for each approach.

However, different from the analogic workflow, the present approach can be performed, associated with an easy transfer process and a reduced number of clinical steps. In addition, adequate restorative materials, implants' system, and patient characteristics should be considered during the treatment plan [17]. Despite the reduced number of clinical steps, this digital workflow requires a fully collaborative and digitally equipped dental laboratory, increasing the lab costs and planning time.

\section{Conclusions}

This dental technique describes the fabrication and use of a hybrid surgical guide custom scanning device to be used during the surgical and prosthetic steps. During the rehabilitation of an edentulous maxilla with dental implants, it was possible to follow the implants' positioning plan during surgery, record the maxillomandibular relationship, and transfer the implants' position to manufacture the maxillary full-arch implant-supported prosthesis. This dental technique provides a digital workflow protocol using a reduced number of clinical steps, dental office visits, and number of devices that need be manufactured by the dental technician. 
Author Contributions: Conceptualization, F.F.T., M.M.H., L.R.G., and J.P.M.T.; methodology, F.F.T., M.M.H., and L.R.G.; investigation, M.A.B., and J.P.M.T.; resources, F.F.T., M.M.H., L.R.G., M.A.B., and J.P.M.T.; writing—original draft preparation, F.F.T., M.M.H., and L.R.G.; writing—review and editing, M.A.B., and J.P.M.T.; visualization, F.F.T., and J.P.M.T.; supervision, F.F.T., M.A.B., and J.P.M.T.; project administration, F.F.T., and J.P.M.T. All authors have read and agreed to the published version of the manuscript.

Funding: This research received no external funding.

Institutional Review Board Statement: Not applicable.

Informed Consent Statement: Informed consent was obtained from all subjects involved in the study.

Data Availability Statement: Not applicable.

Conflicts of Interest: The authors declare no conflict of interest.

\section{References}

1. Michelinakis, G.; Apostolakis, D.; Kamposiora, P.; Papavasiliou, G.; Özcan, M. The direct digital workflow in fixed implant prosthodontics: A narrative review. BMC Oral Health 2021, 21, 37. [CrossRef] [PubMed]

2. Pérez-Giugovaz, M.G.; Park, S.H.; Revilla-León, M. 3D virtual patient representation for guiding a maxillary overdenture fabrication: A dental technique. J. Prosthodont. 2021, 30, 636-641. [CrossRef] [PubMed]

3. Arcas, L.P.B.; Tribst, J.P.M.; Baroudi, K.; Amaral, M.; da Silva-Concílio, L.R.; Vitti, R.P. Dimensional accuracy comparison of physical models generated by digital impression/3D-printing or analog impression/plaster methods. Int. J. Odontostomatol. 2021, 15, 562-568. [CrossRef]

4. Griseto, N.T.; Gallucci, G.O. Digital maxillomandibular relationship registration for an edentulous maxilla: A dental technique. J. Prosthet. Dent. 2021, 125, 858-861. [CrossRef] [PubMed]

5. Moura, G.F.; Siqueira, R.; Meirelles, L.; Maska, B.; Wang, H.-L.; Mendonça, G. Denture scanning technique for computer-guided implant-supported restoration treatment of edentulous patients. J. Prosthet. Dent. 2021, 125, 726-731. [CrossRef] [PubMed]

6. Mai, H.-N.; Lee, D.-H. A digital technique to replicate edentulous arches with functional borders and accurate maxillomandibular relationship for digital complete denture. J. Prosthodont. 2020, 29, 356-359. [CrossRef] [PubMed]

7. Flügge, T.; van der Meer, W.J.; Gonzalez, B.G.; Vach, K.; Wismeijer, D.; Wang, P. The accuracy of different dental impression techniques for implant-supported dental prostheses: A systematic review and meta-analysis. Clin. Oral Implants Res. 2018, 29 (Suppl. 16), 374-392. [CrossRef] [PubMed]

8. Figueras-Alvarez, O.; Cantó-Navés, O.; Real-Voltas, F.; Roig, M. Protocol for the clinical assessment of passive fit for multiple implant-supported prostheses: A dental technique. J. Prosthet. Dent. 2020. [CrossRef] [PubMed]

9. Siqueira, R.A.C.; Cabral, B.L.; Siqueira, G.R.; Mendonça, G.; Wang, H.L. Using digital technique to obtain the ideal soft tissue contour in immediate implants with provisionalization. Implant Dent. 2019, 28, 411-416. [CrossRef] [PubMed]

10. Bover-Ramos, F.; Viña-Almunia, J.; Cervera-Ballester, J.; Peñarrocha-Diago, M.; García-Mira, B. Accuracy of implant placement with computer-guided surgery: A systematic review and meta-analysis comparing cadaver, clinical, and in vitro studies. Int. J. Oral Maxillofac. Implant 2018, 33, 101-115. [CrossRef] [PubMed]

11. Li, J.; Galli, M.; Chen, Z.; Venezia, P.; Mangano, F.; Lepidi, L. A novel digital technique for maintaining maxillomandibular relations in fixed prosthetic rehabilitations. J. Dent. 2021, 114, 103798. [CrossRef] [PubMed]

12. Tribst, J.P.M.; de Oliveira Dal Piva, A.M.; da Silva-Concílio, L.R.; Ausiello, P.; Kalman, L. Influence of implant-abutment contact surfaces and prosthetic screw tightening on the stress concentration, fatigue life and microgap formation: A finite element analysis. Oral 2021, 1, 88-101. [CrossRef]

13. Michelinakis, G.; Nikolidakis, D. Using the surgical guide for impression-free digital bite registration in the edentulous maxilla-a technical note. Int. J. Implant Dent. 2019, 5, 19. [CrossRef] [PubMed]

14. Yang, J.-W.; Liu, Q.; Yue, Z.-G.; Hou, J.-X.; Afrashtehfar, K.I. Digital workflow for full-arch immediate implant placement using a stackable surgical guide fabricated using SLM technology. J. Prosthodont. 2021, 30, 645-650. [CrossRef] [PubMed]

15. Dahiya, A.; Garbacea, A.; Kattadiyil, M.T.; AlHelal, A. Digital technology for performing a nasopalatine and greater palatine nerve block with a modified implant surgical guide: A technique article. J. Prosthet. Dent. 2018, 120, 338-342. [CrossRef] [PubMed]

16. Turkyilmaz, I. Keys to achieving successful restoratively driven implant placement with CAD/CAM surgical guide: A technical note. J. Stomatol. Oral Maxillofac. Surg. 2019, 120, 462-466. [CrossRef] [PubMed]

17. Datte, C.E.; Datte, F.B.; Borges, A.L.; Campos, J.F.; Lopes, G.D.; Tribst, J.P.; Nishioka, R.S. The influence of restorative material, bone height and implant system on the stress distribution of implant-supported posterior crowns. Int. J. Dev. Res. 2021, 11, 1-7. 